\title{
Refined atrial fibrillation screening and cost- effectiveness in the German population
}

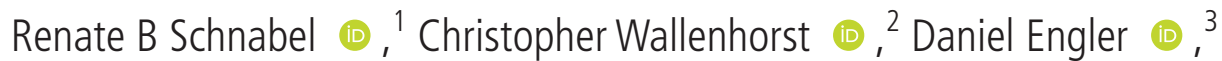 \\ Stefan Blankenberg, ${ }_{1}^{4}$ Norbert Pfeiffer, ${ }^{5}$ Ngoc Anh Spruenker, ${ }^{6}$ Matthias Buettner, ${ }^{7}$ \\ Matthias Michal, ${ }_{1}^{8}$ Karl J Lackner, ${ }_{1}^{9}$ Thomas Münzel, ${ }^{10}$ Philipp S Wild, ${ }_{1}^{11}$ \\ Carlos Martinez 이, ${ }^{2}$ Ben Freedman $(1),{ }^{12}$ Gutenberg Health Study investigators
}

\begin{abstract}
- Additional supplemental material is published online only. To view, please visit the journal online (http://dx.doi. org/10.1136/heartjnl-2020318882)
\end{abstract}

For numbered affiliations see end of article.

Correspondence to Dr Renate B Schnabel, Cardiology, University Heart Center Hamburg, Hamburg 52 20246, Germany; r.schnabel@uke.de

Received 22 December 2020 Accepted 11 June 2021

\section{Check for updates}

(c) Author(s) (or their employer(s)) 2021. Re-use permitted under CC BY-NC. No commercial re-use. See rights and permissions. Published by BMJ.

To cite: Schnabel RB Wallenhorst C, Engler D, et al. Heart Epub ahead of print: [please include Day Month Year]. doi:10.1136/ heartjnl-2020-318882

\section{ABSTRACT \\ Objective Little is known on optimal screening population for detecting new atrial fibrillation (AF) in the community. We describe characteristics and estimate cost-effectiveness for a single timepoint electrocardiographic screening.}

Methods We performed a 12-lead ECG in the German population-based Gutenberg Health Study between 2007 and $2012(n=15010)$, mean age $55 \pm 11$ years, $51 \%$ men and collected more than 120 clinical and biomarker variables, including $\mathrm{N}$-terminal pro B-type natriuretic peptide (Nt-proBNP), risk factors, disease symptoms and echocardiographic variables.

Results Of 15010 individuals, 466 (3.1\%) had AF. New AF was found in 32 individuals, $0.2 \%$ of the total sample, $0.5 \%$ of individuals aged $65-74$ years and predominantly men (86\%). The classical risk factor burden was high in individuals with new AF. The median estimated stroke risk was 2.2\%/year, while risk of developing heart failure was $21 \%$ over 10 years. In the 65-74year age group, the cost per quality-adjusted life-year gained resulting from a single timepoint screening was $€ 30361$. In simulations, the costs were highly sensitive to AF detection rates, proportion of treatment and type of oral anticoagulant. Prescreening by Nt-proBNP measurements was not cost-effective in the current setting.

Conclusions In our middle-aged population cohort, we identified $0.2 \%$ new AF by single timepoint screening. There was a significant estimated risk of stroke and heart failure in these individuals. Cost-effectiveness for screening may be reached in individuals aged 65 years and older. The simple age cut-off is not improved by using Nt-proBNP as a biomarker to guide a screening programme.

\section{INTRODUCTION}

Atrial fibrillation (AF) is the most common arrhythmia of clinical importance in the general population worldwide. ${ }^{1}$ Its prevalence increases with age and is higher in men. ${ }^{2}$ In contrast to the imminent AF epidemic in our society and costly treatments of the disease and its consequences, large-scale screening efforts have remained scant and the ideal target group in the general population is unknown. ${ }^{34}$

In a recent study, we found that unknown $\mathrm{AF}$ was detected in $1.5 \%$ on a single screen using an inexpensive rapid readout device, which was found likely to be cost-effective for stroke prevention in individuals aged 65 years or older (SEARCH-AF). ${ }^{5}$ An individual patient meta-analysis of more than 140000 screened subjects suggested a detection rate of $1.4 \%$ in those aged $\geq 65$ years, with a continuous gradation in detection rate with each 5 years of age. ${ }^{6}$ However, little is known on the screening effectiveness in the middle-aged general European population (35-74 years), where the prevalence of $\mathrm{AF}$ is about $2.5 \%$ ? $^{7}$

\section{METHODS}

\section{Study participants}

The population-based study cohort constitutes a random sample of individuals from the German region of Mainz and Mainz-Bingen (Gutenberg Health Study (GHS) cohort enrolled from 2007 to 2012). Study participants were aged $35-74$ years at enrolment and invited by letter within 10-year age strata. Comprehensive information on cardiovascular risk factors were collected during a 5-hour study visit by standardised computer-assisted interview, anthropometric measurements, non-invasive cardiovascular function testing and transthoracic echocardiography (for further details, see the online supplemental methods). Medication information was collected from the packages brought by the participants. ${ }^{8}$ Exclusion of individuals based on the following conditions: (1) individuals with missing information on AF history and missing study ECG, (2) individuals with missing information on $\mathrm{AF}$ history and without AF in the baseline ECG and (3) individuals without AF history with missing ECG at baseline. N-terminal pro B-type natriuretic peptide (Nt-proBNP) was measured on the ELECSYS 2010 platform using an electrochemiluminescence immunoassay (Roche Diagnostics).

\section{Outcome}

A 10 s 12-lead ECG was recorded for every participant (GE Cardiosoft). We diagnosed AF based on a history of AF reported by the participant and/or the ECG documentation of AF or atrial flutter. ${ }^{9} \mathrm{AF}$ was adjudicated by at least two physicians with cardiology training and experience in ECG reading. In difficult cases, a third opinion by an electrophysiologist was obtained. Screening-detected AF or new AF was defined as AF on the study ECG in 
individuals without a history of AF. Individuals with new AF on the study ECG were redefined as known AF if they were taking the following medications: oral anticoagulant (OAC), digoxin or Vaughan Williams class I or III antiarrhythmic drugs. ${ }^{10}$

Prior to enrolment, participants provided written, informed consent. All authors have read and approved the manuscript as written.

\section{Statistical methods}

To assess disease risk, we used a published risk algorithm to estimate predicted AF risk in individuals with prevalent $\mathrm{AF}^{11}{ }^{11}$ To understand stroke risk, we calculated the $\mathrm{CHA}_{2} \mathrm{DS}_{2}$-VASc (heart failure, hypertension, age $\geq 75$ years (double score), diabetes, prior stroke or transient ischemic attack (TIA; double score), vascular disease, age 65-74 years, sex class (female)) score adding together the points for the following conditions: congestive heart failure/left ventricular dysfunction (1), high blood pressure (1), age $>75$ years (2), diabetes (1), stroke/transient ischaemic attack/thromboembolism (2), vascular disease (coronary artery disease, myocardial infarction, peripheral artery disease and aortic plaque) (1), age 65-74 years (1) and sex category female (1). The estimated stroke risk was also adjusted for warfarin intake assuming that warfarin provides a reduction in thromboembolism events. ${ }^{2}$ For the estimation of heart failure risk and risk of stroke or death, respective risk algorithms from the literature were applied. ${ }^{1112}$

In further analyses, we used random forest machine learning algorithms to identify clinical and biomarker variables most strongly related with $\mathrm{AF}$ among 121 variables available in the GHS. ${ }^{13}$ Least absolute shrinkage and selection operator (LASSO) and forward selection methods were applied to understand the validity of the results of the machine learning algorithm. We used all AF cases in order to increase the number of response variables with an expected relatively small number of cases in the cohort. Missing values were imputed as the weighted average of the non-missing observations using weights determined from random forests (for details, please see the online supplemental methods). ${ }^{14}$ If variable values were missing in more than $70 \%$ of individuals, the respective variable was not used for analyses. We used $70 \%$ as a cut-off in order to accommodate biomarker measurements which were available in a subgroup accepting the introduction of potential bias. For clinical variables, we assumed missingness at random.

The complete data sample was split into a training and a test (validation) data set before any statistical analysis was carried out. All individuals in the complete data sample were randomly allocated to the data sets using a ratio of $3: 2$ individuals. The fit of each statistical method (generated in the training data set) was validated by applying the fit to the test set. The performance of each method was presented by the C-statistic for the random forest method or area under curve (AUC) for the LASSO and forward selection method. ${ }^{15} 16$ Sensitivity and specificity analyses were performed for the best discriminator selected (Nt-proBNP), and the number needed to screen to find one new AF case depending on various Nt-proBNP cut-offs was calculated. The $\mathrm{Nt}$-proBNP cut-off that maximised the sum of sensitivity and specificity, giving the sensitivity function a $20 \%$ higher weight, was identified.

\section{Cost-effectiveness analyses}

A cost-effectiveness analysis to understand the impact on healthcare expenditure for a single timepoint electrocardiographic screening was performed, comparing the scenarios with and without screening for AF in the German population aged 65-74 years based on Markov models that accounted for OAC treatment, incident strokes, major bleeds and all-cause mortality. ${ }^{5}$ The target variables of the cost-effectiveness analyses were costs per quality-adjusted life-year (QALY) gained and costs per stroke prevented.

In addition, refined screening scenarios (other than age cutoff (65 years) alone) were assessed by using an age cut-off plus clinical AF risk score based threshold of 5\% and 10\%, and age cut-off plus a point of care testing of Nt-proBNP to preselect individuals at higher risk of AF before performing a 12-lead ECG.

For cost-effectiveness analyses we used Stata MP V.14.2 (StataCorp LLC) and for all other analyses R V.3.4.1 (R Foundation for Statistical Computing).

\section{Patients and public involvement}

Patients and public were not involved in the design, conduct, reporting or dissemination plans of our research.

\section{RESULTS}

After excluding $72(0.5 \%)$ individuals due to missing information on the history of AF from the overall GHS sample $(n=15010$; $100 \%)$, we assessed $466(3.1 \%)$ individuals with prevalent AF. New AF comprised $32(0.2 \%)$ individuals. The GHS weighted (sex, age and region) prevalence of AF was 2.5\% $(n=380)$, including $0.2 \%(n=25)$ prevalence of new AF.

Table 1 and online supplemental table 1 summarise the characteristics of the study sample by AF status. The new AF group revealed the lowest proportion of women (14.1\%). A high classical risk factor burden was seen in individuals with both new AF and known AF. Symptoms of dyspnoea and palpitations were about three times more common in known $\mathrm{AF}$ and almost twice as frequent in unknown AF, as in individuals without AF. The biomarker Nt-proBNP was about 14 times higher in the new $\mathrm{AF}$ group, and about four times higher in known AF compared with no AF.

Figure 1 demonstrates that among individuals with AF the relative proportion according to age decade in the subgroups of individuals with known and new AF was similar in both groups rising from less than 10\% (35-44 years) to approximately $60 \%$ of those with AF in the latest age decade of $65-74$ years. In online supplemental figure 1 and online supplemental table 2 , the distribution of predicted risk of AF based on an AF risk algorithm is provided. ${ }^{17}$

The risk of adverse events in new AF cases is shown in figure $2 \mathrm{~A}-\mathrm{C}$. The median $\mathrm{CHA}_{2} \mathrm{DS}_{2}$-VASc score was two with a median annual stroke risk of $2.2 \%$. The distribution of stroke risk by $\mathrm{CHA}_{2} \mathrm{DS}_{2}-\mathrm{VASc}$ score in new $\mathrm{AF}$ cases is provided in online supplemental figure 2 and figure $2 \mathrm{~A}$. The 10 -year risk of developing heart failure was $21 \%$ (25th/75th percentile $11 / 37 \%$ ) shown in figure $2 \mathrm{C}$, and the 5 -year risk of stroke or death was $30 \%$ (25th/75th percentile 19/44\%) for individuals with new AF (figure 2B).

In random forest selection, Nt-proBNP was the single variable most strongly related to prevalent $\mathrm{AF}$ validated by stepwise selection and LASSO methods (online supplemental figure 3). The model fit C-statistic was 0.829 for the random forest selection, the AUCs 0.802 for the stepwise selection and 0.834 for the LASSO methods. Evaluating different Nt-proBNP cut-offs with respect to sensitivity and specificity of high AF risk prediction revealed an optimal clinical threshold value of $120 \mathrm{ng} / \mathrm{L}$, that is the 77 th percentile of Nt-proBNP concentrations, with 
Table 1 Characteristics of study participants by AF status weighted for age and sex of the population in Mainz and Mainz-Bingen area (weighted $\mathrm{n}=14937$ )

\begin{tabular}{|c|c|c|c|}
\hline & No $A F(n=14557)$ & Known AF $(n=355)$ & New AF $(n=25)$ \\
\hline Age, years & $52.3 \pm 11.0$ & $63.2 \pm 9.5$ & $64.9 \pm 9.1$ \\
\hline Female, \% & $50.6(49.8-51.4)$ & $34.4(29.5-39.3)$ & $14.1(0.5-27.7)$ \\
\hline Current smoking, $\%$ & $20.9(20.2-21.6)$ & $13.4(9.9-17.0)$ & $24.7(7.8-41.6)$ \\
\hline Body mass index, $\mathrm{kg} / \mathrm{m}^{2}$ & $26.4(23.7 / 29.8)$ & $28.4(25.9 / 32.0)$ & $27.4(26.1 / 33.4)$ \\
\hline Systolic blood pressure, $\mathrm{mm} \mathrm{Hg}$ & $128(118 / 139)$ & $131(120 / 142)$ & $136(120 / 147)$ \\
\hline Diastolic blood pressure, $\mathrm{mm} \mathrm{Hg}$ & $82(76 / 88)$ & $80(74 / 87)$ & $84(77 / 92)$ \\
\hline Heart rate, bpm & $69(62 / 75)$ & $67(58 / 76)$ & $77(68 / 88)$ \\
\hline Diabetes, $\%$ & $6.0(5.6-6.4)$ & $14.5(10.8-18.2)$ & $23.6(7.0-40.3)$ \\
\hline Hypertension, \% & $44.5(43.7-45.3)$ & $70.1(65.3-74.9)$ & $66.7(48.2-85.2)$ \\
\hline Dyslipidaemia, \% & $27.7(27.0-28.4)$ & $43.7(38.5-48.9)$ & $30.5(12.5-48.6)$ \\
\hline Prevalent myocardial infarction, \% & $2.1(1.9-2.3)$ & $14.7(11.0-18.4)$ & $6.4(0-16.0)$ \\
\hline Prevalent stroke, \% & $1.4(1.2-1.6)$ & $8.7(5.8-11.6)$ & 0 \\
\hline Heart failure, $\%$ & $16.4(15.8-17.0)$ & $44.3(39.1-49.5)$ & $41.7(22.4-61.0)$ \\
\hline Dyspnoea, \% & $10.7(10.2-11.2)$ & $30.8(26.0-35.6)$ & $22.1(5.8-38.4)$ \\
\hline Palpitations, \% & $15.2(14.6-15.8)$ & $46.5(41.3-51.7)$ & $25.9(8.7-43.1)$ \\
\hline NT-proBNP (pmol/L)* & $61(31 / 115)$ & $261(80 / 948)$ & $868(276 / 1951)$ \\
\hline Thyroid-stimulating hormone (mU/L) & $1.04(0.73 / 1.47)$ & $1.0(0.7 / 1.4)$ & $1.06(0.64 / 1.28)$ \\
\hline $\mathrm{C}$ reactive protein $(\mathrm{mg} / \mathrm{L})$ & $1.5(0.5 / 3.1)$ & $2.1(1.0 / 4.2)$ & $2.9(1.2 / 4.3)$ \\
\hline
\end{tabular}

Mean values and SD for continuous variables, median and 25th/75th percentiles for skewed continuous variables, or per cent and a $95 \% \mathrm{Cl}$ for categorical variables.

${ }^{*}$ Available for the first 5000 study participants.

$\mathrm{AF}$, atrial fibrillation; Nt-proBNP, N-terminal pro B-type natriuretic peptide.

a number needed to screen of 13 , a test sensitivity of 0.68 and a test specificity of 0.78 (online supplemental table 3 and online supplemental figure 4).

\section{Cost-effectiveness}

We estimated costs per QALY gained by single timepoint AF screening in those aged 65-74 years of $€ 30361$ for a prevalence of new AF of $0.5 \%$ and VKA prescriptions in $58 \%$ of cases. The costs per stroke prevented were inversely related to the prevalence of new AF (figure 3). Thus screening in a younger age group with both a lower prevalence of new AF and a lower estimated stroke risk would not be cost-effective. A higher proportion taking $\mathrm{OAC}$ therapy reduced the costs per stroke prevented. With a higher proportion of OAC uptake, the distance between the curves for different proportions of AF prevalence declined and the additional decrease in costs was less pronounced (figure 3). A sensitivity analysis demonstrated that a higher

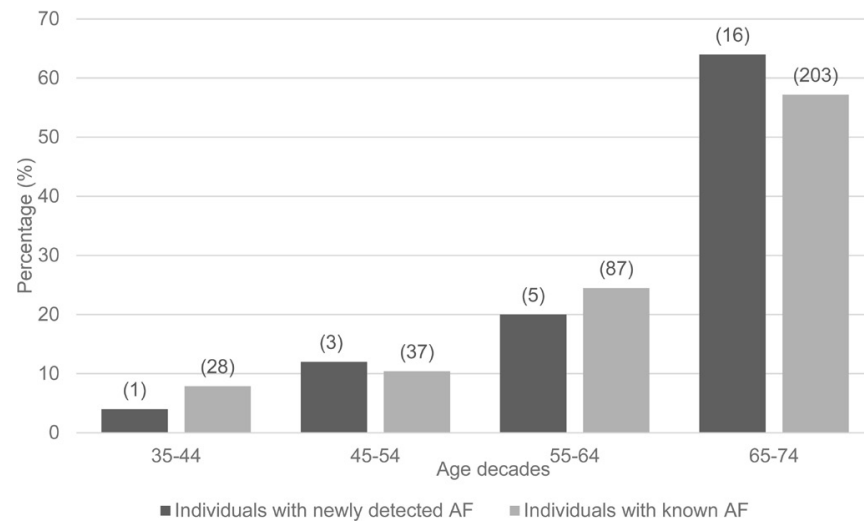

Figure 1 Distribution of individuals with known $A F(n=355)$ and new detected $A F(n=25)$ by age decades, weighted for residence, age and sex of the population in Mainz and Mainz-Bingen area $(n=14937)$. AF, atrial fibrillation. proportion of non-vitamin $\mathrm{K}$ oral anticoagulant (NOAC) intake compared with VKA increased the costs per prevented stroke (figure 4).

The number of strokes avoided and costs per QALY gained using a stepwise screening approach including Nt-proBNP prescreening tests are provided in online supplemental table 4 and online supplemental figures 5,6. As an example, using an $\mathrm{Nt}$-proBNP threshold of $120 \mathrm{ng} / \mathrm{L}, 23 \%$ of the prescreened individuals get a positive AF test result and hence a subsequent ECG. In the German population, this would lead to 17499 individuals with newly identified AF and subsequently 763 strokes avoided according to OAC treatment. The costs per stroke prevented would be $€ 140800$ and the costs per QALY gained $€ 36382$. Independent of the threshold value, Nt-proBNP prescreening was less cost-effective for stroke prevention or QALYs gained than the ECG screening using age alone. When using a clinical risk score guided screening using a $5 \%$ or $10 \%$ risk cut-off, $\mathrm{n}=972$ (sensitivity $86.7 \%$ (online supplemental table 5)) and $\mathrm{n}=539$ (sensitivity 48.1\%) strokes would have been avoided, respectively.

\section{DISCUSSION}

In a large, middle-aged to older population-based cohort of 15010 individuals, we identified $32(0.2 \%)$ new AF cases by a single ECG. The yield of newly identified AF increased with age and risk factor burden.

The definition of the most efficient method of screening for $\mathrm{AF}$ in the ageing population is an urgent research and health economics question. ${ }^{4}$ In our study, undiagnosed AF was not necessarily silent. One quarter of new AF individuals indicated that they had the typical symptom of palpitations and one-fifth had dyspnoea, but despite that, patients had not been diagnosed. The heart rate of participants with new AF has shown a broad range. ${ }^{45}$ In our sample, mean heart rate was higher compared with individuals with known disease or participants without AF but was still below 80 , which may mean that symptoms were less 

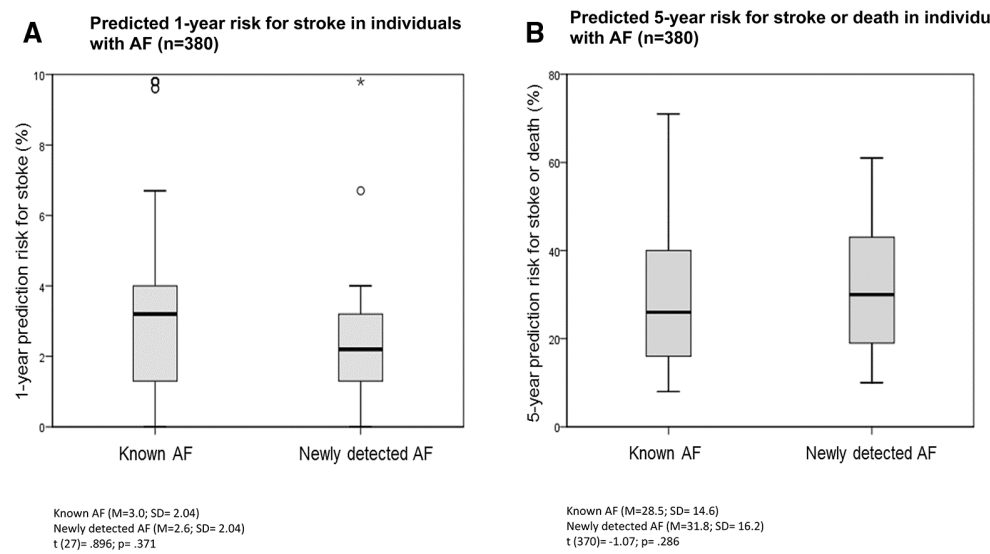
Predicted 10-year risk for heart failure in individuals
with AF ( $n=380)$

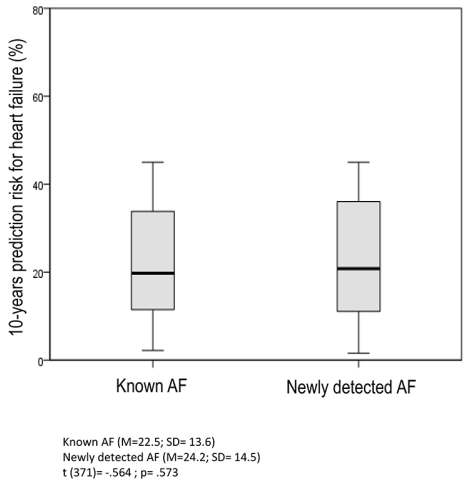

Figure 2 Boxplots of predicted risk of stroke (A), stroke or death (B) and heart failure (C) in individuals with known and newly detected AF. Risk algorithms for annual stroke risk ( $\mathrm{CHA}_{2} \mathrm{DS}_{2}$-VASc score) ${ }_{1}^{11} 10$-year heart failure risk ${ }^{30}$ and 5 -year risk of stroke or death ${ }^{12}$ were used. Heart failure risk scores were truncated at the upper risk estimate of $\geq 45 \%$ to avoid inaccuracies due to extreme observations. Stroke risk assessed by $\mathrm{CHA}_{2} \mathrm{DS}_{2}-\mathrm{VASC}$ score is adjusted for warfarin intake. Cardiac murmur is not available in the Gutenberg Health Study and is left out of the calculation of heart failure risk. Data are weighted for residence, age and sex of the population in Mainz and in Mainz-Bingen area. AF, atrial fibrillation.

notable, and consistent with not seeking medical attention for the symptom. These signs and symptoms are not specific for AF, for example, $15 \%$ of individuals without diagnosed $\mathrm{AF}$ reported palpitations but may be sensitive and thus help to guide a closer look and possibly more intensified diagnostics based on symptoms alone.

Classical AF risk factors were more common in individuals with new AF. Strikingly, about $40 \%$ of new AF participants had heart failure based on symptoms and echocardiographic findings. Echocardiography certainly is not a screening tool at the population level; however, our data confirm the importance of heart failure as a risk factor for AF not only in the clinical setting. ${ }^{18}$ When applying a recent risk prediction algorithm, ${ }^{11}$ the median risk of $\mathrm{AF}$ in individuals with incidentally diagnosed $\mathrm{AF}$ was highest; however, with a median of $6.7 \%$, predicted 10 -year risk was still comparatively low and therefore may not be expected to be a very useful guide to screening. The calculated risk in participants without AF was negligible. These findings indicate the remaining limitation of the scoring system to accurately identify individuals at risk of AF and call for improvement of prediction tools.

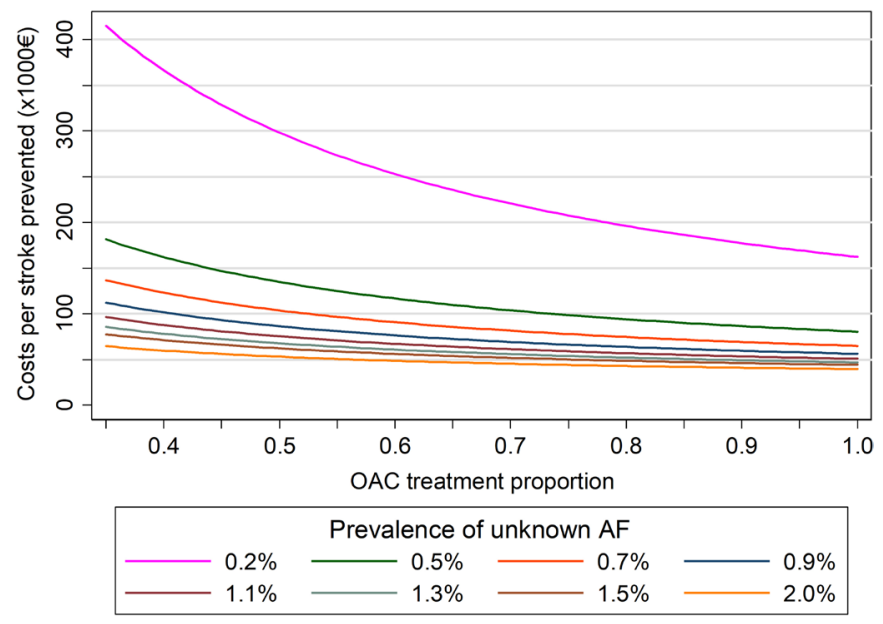

Figure 3 Costs per stroke prevented by OAC intake stratified by the prevalence of unknown $A F$. $A F$, atrial fibrillation; $O A C$, oral anticoagulant.
Overall, age remained the major determinant of risk of unknown $\mathrm{AF}$ and is certainly the easiest variable to select patients for screening. This is the basis of guideline recommendation for opportunistic screening for AF in individuals aged $\geq 65$ years. ${ }^{192}$ The risk of adverse events in individuals with first detected AF was not negligible: the median $\mathrm{CHA}_{2} \mathrm{DS}_{2}$-VASc score was 2 , only $31 \%$ had a score of $<2$. Respectively, $69 \%$ of individuals with new $\mathrm{AF}$ had a score of $\geq 2$ and would be eligible for an OAC prescription according to guidelines. ${ }^{20}$ Among individuals with known $\mathrm{AF}, 75 \%$ had a $\mathrm{CHA}_{2} \mathrm{DS}_{2}$-VASc score of $\geq 2$; however, only $35 \%$ were taking an OAC. A $\mathrm{CHA}_{2} \mathrm{DS}_{2}$-VASc score that requires the evaluation of therapeutic anticoagulation in individuals with first detected $\mathrm{AF}$ in community screening has been reported earlier. ${ }^{41721}$

Our data further support the need to raise the awareness for AF. We and others have shown that a relevant proportion of individuals did not know their very likely diagnosis of $\mathrm{AF}^{5}{ }^{22}$ They were older, more often men, had diabetes and present

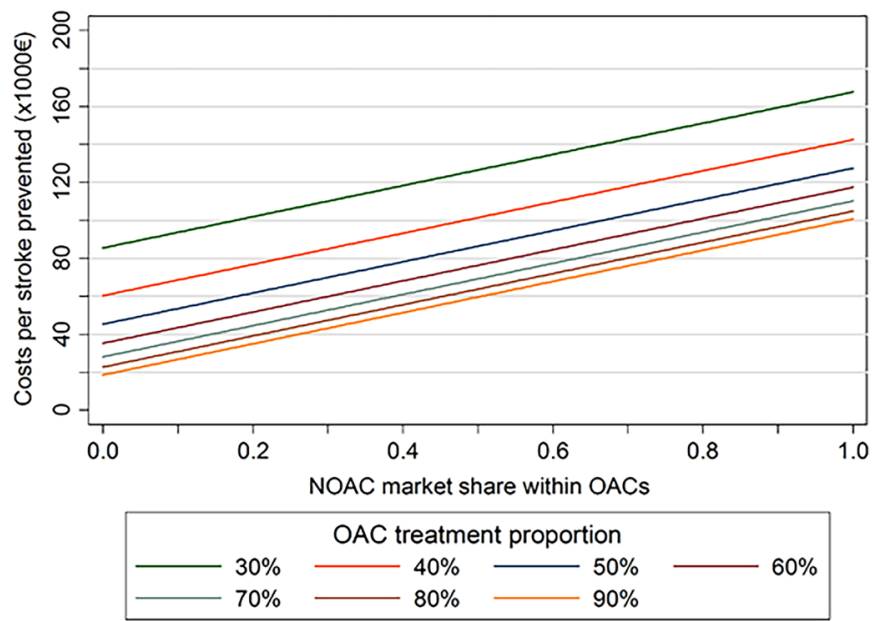

Figure 4 Costs per stroke prevented in relation to the proportion of NOAC versus VKA stratified by the proportion of OAC intake. AF, atrial fibrillation; NOAC, non-vitamin $\mathrm{K}$ oral anticoagulant; OAC, oral anticoagulant. 
higher values of NT-proBNP, thyroid-stimulating hormone and $\mathrm{C}$ reactive protein.

\section{Cost-effectiveness}

The proportion of known and new AF cases increased similarly with age, while the absolute numbers in the first two age decades in both groups remained small. The proportion of OAC uptake affects cost-effectiveness especially for the OAC proportion band below $65 \%$. Another cost driver was NOAC treatment compared with VKA. For an OAC uptake of more than $90 \%$, which has been shown in the STROKESTOP study, ${ }^{23}$ treatment with NOAC only compared with VKA only revealed a fivefold increase in costs per stroke prevented, from about $€ 20000$ to $€ 100000$. These differences may become less pronounced once NOAC patents run out. Overall, cost-effectiveness for a single timepoint screening appears to be given in age groups 65 years and older. ${ }^{5}$

We restricted cost-effectiveness analyses to participants 65 years or older due to the screening yield of unknown $\mathrm{AF}$ in the sample and risk factor burden that warrants anticoagulation. However, the prevalence of new AF of $0.5 \%$ after the age of 65 years in this German cohort with an upper age limit of 74 years at enrolment remained low. In other screening studies such as SEARCH-AF, ${ }^{5}$ which also included individuals aged 65 years and older (mean age $79 \pm 6$ years) the $\mathrm{AF}$ detection rate was $1.4 \%$. A meta-analysis of single timepoint screening confirmed a detection rate of $1.4 \%$ in individuals $\geq 65$ years. ${ }^{24}$ As shown by our simulations, cost-effectiveness is highly dependent on the prevalence of undetected or undertreated $\mathrm{AF}$ in the screened population.

The search for more specific and sensitive biomarkers for AF in addition to the CHARGE-AF equation was one of the motivations for the GHS. We assumed that elevated natriuretic peptide concentrations mirror underlying cardiac stress as a predisposition to the arrhythmia or episodes of the AF, which increase biomarker secretion and confirmed that Nt-proBNP was the best biomarker. This assumption has also been the rationale for the use of this biomarker in STROKESTOP-2 study, as it was hypothesised the biomarker would be more cost-effective than a simple age criterion as in the STROKESTOP-1 study and in most other current and proposed screening studies. A similar biomarker threshold of Nt-proBNP (125 pmol/L) identified fewer individuals with AF in the STROKESTOP-2 study, which may be explained by an older study cohort with possibly more comorbidities, overall higher Nt-proBNP concentrations and a lower discriminatory ability. ${ }^{22}$ In our cohort, we found that the strategy of refined screening by upfront application of current clinical risk scores or Nt-proBNP measurement was unlikely to be more cost-effective for a single timepoint screening at present. Cost-effectiveness requires more specific markers than Nt-proBNP and/or lower costs for the test to be applied at scale. In our sample, the costs per QALY achieved using Nt-proBNP prescreening would have been equal to the ECG screening only if the test itself cost $€ 6.23$. Lower costs, which are likely in the future, could render biomarker-guided prescreening cost-effective.

\section{Limitations}

Despite the large cohort size, the number of new AF cases was small and may result in unstable estimates. An older cohort over 75 years, such as in the STROKESTOP studies, might have had a higher yield for screen-detected AF and cost-effectiveness as AF incidence increases with age.
A single 12-lead ECG is not sufficient to rule out prevalent AF, and we underestimate the true disease burden and capture more persistent forms of $\mathrm{AF}^{25}$ However, longer term monitoring or repeat ECG registration may be less feasible at a large scale for screening in the general population and may find $\mathrm{AF}$ with a lower stroke risk as seen in implanted device-detected $\mathrm{AF}^{26}$ More intensified screening is efficient in predefined subgroups at increased risk. Such groups could be defined by age ${ }^{4}$ or by risk factor burden, in particular heart failure, which will increase the yield of new AF. Elevated biomarkers may help guide screening and may reduce the number needed to screen. $^{2}$

Furthermore, the proportion of individuals with known AF on OAC would most likely be higher today since the change of recommendations for anticoagulation towards a lower $\mathrm{CHA}_{2} \mathrm{DS}_{2}$-VASc score, which would render screening more effective. ${ }^{9}$ Furthermore, current guidelines more strongly recommend screening and explicitly recommend screening in patients with hypertension or obstructive sleep apnoea, which may impact screening efficiency. ${ }^{20}$

A limitation of any cost-effectiveness analysis to date is that the results strongly depend on assumed input parameters and outcome information from external studies not designed for screening purposes. Therefore, we investigated the effect of a change in input parameters on the costs per QALY gained and costs per stroke prevented across a range of inputs. Furthermore, the costeffectiveness analysis included simplifications, for example, NOACs were assumed to have the same characteristics as VKAs with respect to treatment persistence and to the incidence of stroke, mortality and major bleeds during treatment, although in practice, NOACs are likely related to a lower risk of stroke, major bleeds and mortality. ${ }^{28}$ These simplifications may have to be revised once more robust reallife data become available.

We conclude that screening for AF by 12-lead ECG in individuals from the general population aged $<65$ years yields a low proportion of new AF cases and is therefore not cost-effective. The costeffectiveness of single timepoint screening improves with increasing age and risk factor burden and when simpler methods for detection are used, such as handheld devices or repeat ECGs as available from smart watches. ${ }^{29}$ At present, a simple age cut-off appeared to be the most effective selection criterion for screening. Our results

\section{Key messages}

What is already known on this subject?

- Individuals with atrial fibrillation (AF) have a high risk of stroke, and opportunistic screening for AF is recommended in patients aged 65 years or older. Data on screening yield and cost-effectiveness are limited.

\section{What might this study add?}

- This population-based study of middle-aged to older individuals shows that cost-effectiveness may be achieved with single time point screening in older individuals with age as the strongest predictor, possibly optimised by clinical risk factors. Prescreening by B-type natriuretic peptide measurement at current costs and precision is not efficient.

How might this impact on clinical practice?

- Age remains the major determinant of screen-detected AF in the population and is easily available. Cost-effectiveness of AF screening may be reached in people 65 years and older for single timepoint screening, especially if the 12-lead ECG could be replaced by, for example, handheld devices. 
may spur future investigations of the characteristics of individuals who are at increased risk of developing AF such as classical risk factors combined in more accurate risk scores and biomarkers, for example, Nt-proBNP and in whom simple screening efforts may be more efficient to detect AF. Such data are needed to implement large-scale screening at the population level and inform public health programmes.

\section{Author affiliations}

'Department of Cardiology, University Heart Center Hamburg, Hamburg, Germany ${ }^{2}$ Institute for Epidemiology, Statistics and Informatics GmbH, Frankfurt, Germany ${ }^{3}$ Department of Cardiology, Universitäres Herzzentrum Hamburg (UHZ), Hamburg, Germany

${ }^{4}$ Clinic for General and Interventional Cardiology, University Heart Center Hamburg, Hamburg, Germany

${ }^{5}$ Department of Ophthalmology, University Medical Center, Johannes Gutenberg University Mainz, Mainz, Germany

${ }^{6}$ University Heart and Vascular Center Hamburg Eppendorf, Hamburg, Germany ${ }^{7}$ Institute of Medical Biostatistics, Epidemiology and Informatics, University Medical Center, Johannes Gutenberg University Mainz, Mainz, Germany

${ }^{8}$ Department of Psychosomatic Medicine and Psychotherapy, University Medical Center of the Johannes Gutenberg-University Mainz, Hamburg, Germany ${ }^{9}$ Institute of Clinical Chemistry and Laboratory Medicine, University Medical Center Mainz, Mainz, Germany

${ }^{10}$ Department of Medicine 2, University Medical Center Mainz, Mainz, Germany ${ }^{11}$ Department of Preventive Cardiology and Preventive Medicine, University Medical Center Mainz, Mainz, Rhineland-Palatinate, Germany

${ }^{12}$ University of Sydney, Charles Perkins Centre, Heart Research Institute, Sydney, New South Wales, Australia

Acknowledgements We would like to thank the participants and dedicated study staff of the Gutenberg Health Study for their generous contribution of time and efforts.

Contributors RS and BF developed the conceptional framework and the design of the study. CW and CM conducted the economic analysis. NAS and DE calculated the epidemiological analysis. RS, BF, CW, CM, NAS and DE interpreted the data. RS and $\mathrm{BF}$ drafted the manuscript, and CW, CM and DE revised the manuscript critically. SB, TM, PSW, NP, MB and MM commented and approved the version of the manuscript to be published.

Funding The Gutenberg Health Study is funded through the government of Rhineland-Palatinate ('Stiftung Rheinland-Pfalz für Innovation', contract AZ 961386261/733), the research programmes 'Wissen schafft Zukunft' and 'CenterCentre for Translational Vascular Biology (CTVB)' of the Johannes Gutenberg-University of Mainz, and its contract with Boehringer Ingelheim and PHILIPS Medical Systems, including an unrestricted grant for the Gutenberg Health Study. PSW is funded by the Federal Ministry of Education and Research (BMBF 01E01503). TM and PSW are PI of the German Centre for Cardiovascular Research (DZHK). This project has received funding from the European Research Council under the European Union's Horizon 2020 research and innovation programme (grant agreement No 648131), from the European Union's Horizon 2020 research and innovation programme under the grant agreement No 847770 (AFFECT-EU) and German Centre for Cardiovascular Research (DZHK e.V.) (81Z1710103); German Ministry of Research and Education (BMBF 01Z×1408A) and ERACoSysMed3 (031L0239) (RBS).

Competing interests $\mathrm{CW}$ and $\mathrm{CM}$ and employees of the Institute for Epidemiology, Statistics and Informatics GmbH. The Institute has received grants from Bristol-Myers Squibb and Pfizer, and CSL Behring outside the submitted work. BF reports prior fees and advisory board honoraria from Bayer Pharma AG, Boehringer Ingelheim, Daiichi-Sankyo, Omron and Pfizer/BMS and grants to the institution for investigator-initiated studies from BMS/Pfizer. RBS has received speaker honoraria from Bristol-Myers Squibb/Pfizer. MB received lecture fees from Lilly and Takeda, all outside of the submitted work.

Patient consent for publication Not required.

Ethics approval This study was approved by the local ethics committee.

Provenance and peer review Not commissioned; externally peer reviewed.

Data availability statement All data relevant to the study are included in the article or uploaded as supplementary information.

Supplemental material This content has been supplied by the author(s). It has not been vetted by BMJ Publishing Group Limited (BMJ) and may not have been peer-reviewed. Any opinions or recommendations discussed are solely those of the author(s) and are not endorsed by BMJ. BMJ disclaims all liability and responsibility arising from any reliance placed on the content. Where the content includes any translated material, BMJ does not warrant the accuracy and reliability of the translations (including but not limited to local regulations, clinical guidelines, terminology, drug names and drug dosages), and is not responsible for any error and/or omissions arising from translation and adaptation or otherwise.

Open access This is an open access article distributed in accordance with the Creative Commons Attribution Non Commercial (CC BY-NC 4.0) license, which permits others to distribute, remix, adapt, build upon this work non-commercially, and license their derivative works on different terms, provided the original work is properly cited, appropriate credit is given, any changes made indicated, and the use is non-commercial. See: http://creativecommons.org/licenses/by-nc/4.0/.

\section{ORCID iDs}

Renate B Schnabel http://orcid.org/0000-0001-7170-9509

Christopher Wallenhorst http://orcid.org/0000-0001-7944-7347

Daniel Engler http://orcid.org/0000-0002-9519-6258

Carlos Martinez http://orcid.org/0000-0001-6498-6428

Ben Freedman http://orcid.org/0000-0002-3809-2911

\section{REFERENCES}

1 Chugh SS, Havmoeller R, Narayanan K, et al. Worldwide epidemiology of atrial fibrillation. Circulation 2014;129:837-47.

2 Di Carlo A, Bellino L, Consoli D, et al. Prevalence of atrial fibrillation in the Italian elderly population and projections from 2020 to 2060 for Italy and the European Union: the FAI project. Europace2019;21:1468-75.

3 Fitzmaurice DA, Hobbs FDR, Jowett $S$, et al. Screening versus routine practice in detection of atrial fibrillation in patients aged 65 or over: cluster randomised controlled trial. BMJ 2007;335:383.

4 Engdahl J, Andersson L, Mirskaya M, et al. Stepwise screening of atrial fibrillation in a 75-year-old population. Circulation 2013;127:930-7.

5 Lowres N, Neubeck L, Salkeld G, et al. Feasibility and cost-effectiveness of stroke prevention through community screening for atrial fibrillation using iPhone ECG in pharmacies. The SEARCH-AF study. Thromb Haemost 2014;111:1167-76.

6 Lowres N, Olivier J, Chao T-F, et al. Estimated stroke risk, yield, and number needed to screen for atrial fibrillation detected through single time screening: a multicountry patient-level meta-analysis of 141,220 screened individuals. PLoS Med 2019;16:e1002903.

7 Schnabel RB, Wilde S, Wild PS, et al. Atrial fibrillation: its prevalence and risk factor profile in the German general population. Dtsch Arzteb/ Int 2012;109:293-9.

8 Wild PS, Zeller T, Beutel M, et al. Die Gutenberg Gesundheitsstudie. Bundesgesundheitsblatt Gesundheitsforschung Gesundheitsschutz 2012;55:824-30.

9 European Heart Rhythm Association, European Association for Cardio-Thoracic Surgery, Camm AJ, et al. Guidelines for the management of atrial fibrillation: the task force for the management of atrial fibrillation of the European Society of cardiology (ESC). Eur Heart J 2010;31:2369-429.

10 Martinez C, Katholing A, Freedman SB. Adverse prognosis of incidentally detected ambulatory atrial fibrillation. A cohort study. Thromb Haemost 2014;112:276-86.

11 Alonso A, Kriithe BP, Aspelund T, et al. Simple risk model predicts incidence of atria fibrillation in a racially and geographically diverse population: the CHARGE-AF Consortium. J Am Heart Assoc 2013:2.

12 Wang TJ, Massaro JM, Levy D, et al. A risk score for predicting stroke or death in individuals with new-onset atrial fibrillation in the community: the Framingham heart study. JAMA 2003;290:1049-56.

13 Ishwaran H, Kogalur UB, Gorodeski EZ, et al. High-Dimensional variable selection for survival data. J Am Stat Assoc 2010;105:205-17.

14 Breiman L, AJUOC C, Department of Statistics. Setting up, using, and understanding random forests V4. 0, 2003.

15 Friedman J, Hastie T, Tibshirani R. Regularization paths for generalized linear models via coordinate descent. J Stat Softw 2010;33:1

16 Simon N, Friedman J, Hastie T, et al. Regularization paths for COX's proportional hazards model via coordinate descent. J Stat Softw 2011:39:1-13.

17 Deif B, Lowres N, Freedman SB. Screening for atrial fibrillation above age 65 detects an asymptomatic subset at high risk of stroke. Int J Cardiol 2013;164:371-2.

18 Wang TJ, Larson MG, Levy D, et al. Temporal relations of atrial fibrillation and congestive heart failure and their joint influence on mortality. Circulation 2003:107:2920-5.

19 NHFA CSANZ Atrial Fibrillation Guideline Working Group, Brieger D, Amerena J, et al. National heart Foundation of Australia and the cardiac Society of Australia and New Zealand: Australian clinical guidelines for the diagnosis and management of atrial fibrillation 2018. Heart Lung Circ 2018;27:1209-66.

20 Hindricks G, Potpara T, Dagres N, et al. 2020 ESC guidelines for the diagnosis and management of atrial fibrillation developed in collaboration with the European association for Cardio-Thoracic surgery (EACTS). Eur Heart J 2021;42:373-498

21 Tveit $A$, Abdelnoor $M$, Enger $S$, et al. Atrial fibrillation and antithrombotic therapy in a 75-year-old population. Cardiology 2008;109:258-62.

22 Kemp Gudmundsdottir K, Fredriksson T, Svennberg E, et al. Stepwise mass screening for atrial fibrillation using N-terminal B-type natriuretic peptide: the STROKESTOP II study. Europace 2020;22:24-32. 
23 Svennberg E, Engdahl J, Al-Khalili F, et al. Mass screening for untreated atrial fibrillation: the STROKESTOP study. Circulation 2015;131:2176-84.

24 Mant J, Fitzmaurice DA, Hobbs FDR, et al. Accuracy of diagnosing atrial fibrillation on electrocardiogram by primary care practitioners and interpretative diagnostic software: analysis of data from screening for atrial fibrillation in the elderly (SAFE) trial. BMJ 2007:335:380.

25 Nieuwlaat R, Capucci A, Camm AJ, et al. Atrial fibrillation management: a prospective survey in ESC member countries: the Euro heart survey on atrial fibrillation. Eur Heart $J$ 2005:26:2422-34

26 Freedman B, Boriani G, Glotzer TV, et al. Management of atrial high-rate episodes detected by cardiac implanted electronic devices. Nat Rev Cardio/ 2017;14:701-14.
27 Kemp Gudmundsdottir K, Svennberg E, Al-Khalili F, et al. 1359 Mass screening for atrial fibrillation using $n$-terminal pro b-type natriuretic peptide - preliminary results from the strokestop 2 study. Eur Heart J 2018;39:ehy565.

28 Graham DJ, Baro E, Zhang R, et al. Comparative stroke, bleeding, and mortality risks in older Medicare patients treated with oral anticoagulants for nonvalvular atrial fibrillation. Am J Med 2019;132:596-604.

29 Perez MV, Mahaffey KW, Hedlin H, et al. Large-Scale assessment of a Smartwatch to identify atrial fibrillation. N Engl J Med 2019:381:1909-17.

30 Schnabel RB, Rienstra M, Sullivan LM, et al. Risk assessment for incident heart failure in individuals with atrial fibrillation. Eur J Heart Fail 2013;15:843-9. 